\title{
Florística e estrutura de comunidades vegetais em uma cronoseqüiência de Floresta Atlântica no Estado do Paraná, Brasil ${ }^{1}$
}

\author{
Dieter Liebsch ${ }^{2}$, Renato Goldenberg ${ }^{2,3}$ e Márcia Cristina Mendes Marques²
}

Recebido em 29/06/2006. Aceito em 3/04/2007

\begin{abstract}
RESUMO - (Florística e estrutura de comunidades vegetais em uma cronosequiência de Floresta Atlântica no Estado do Paraná, Brasil). Descrevemos a estrutura do estrato arbóreo de três sítios de Floresta Atlântica no litoral do Paraná, objetivando detectar diferenças estruturais gerais e nas populações ao longo do processo sucessional, visando subsidiar futuros planos de recomposição da vegetação. O estudo foi realizado na Reserva Natural Rio Cachoeira, onde foram escolhidos três sítios, cujos históricos indicavam a ocorrência de corte da vegetação há 20 anos, 80 anos e 120 anos. O sítio com 20 anos apresentou os menores valores de riqueza, diversidade, área basal e volume. O sítio com 80 anos apresentou os maiores valores de densidade e riqueza. No sítio com 120 anos foi observado o maior valor de diversidade, equabilidade e volume. Observaram-se algumas diferenças significativas, em termos de diversidade, área basal e volume entre os sítios em diferentes estádios. Comparações das estruturas de populações de espécies que ocorriam em mais de um sítio não mostraram grandes diferenças. Por outro lado, as características das espécies exclusivas de cada sítio influenciaram nas diferenças observadas nos três sítios.
\end{abstract}

Palavras-chave: sucessão secundária, biodiversidade, floresta tropical, conservação

\begin{abstract}
Floristics and structure of plant communities along a chronosequence in the Atlantic Rain Forest of Paraná State, Brazil). We describe the tree structure of three Atlantic Forest fragments on the coast of Paraná, in order to detect differences in community structure throughout the succession process and to support future vegetation restoration projects. The study area was the Reserva Natural Rio Cachoeira, where three sites were chosen based on length of time since the last manmade disturbance (mostly agriculture and lodging); these were, respectively, 20-, 80- and 120-year-old forests. The 20-year-old forest had the lowest richness, diversity, basal area and volume. The 80 -year-old forest had the highest density and richness. The 120-year-old forest had the highest diversity, equitability and volume. Diversity, basal area and volume were significantly different among the three sites. There were no important differences among structures of populations of species shared by the three areas. On the other hand, structural characteristics of exclusive species were important to determine differences among sites.
\end{abstract}

Key words: secondary succession, biodiversity, tropical forest, conservation

\section{Introdução}

A Floresta Atlântica apresenta grande riqueza, tanto animal como vegetal e é considerada um "hotspot" para a conservação da biodiversidade (Myers et al. 2000). Atualmente extremamente fragmentada, a Floresta Atlântica está reduzida a manchas disjuntas, concentradas nas regiões sudeste e sul do Brasil (Leitão Filho 1994), a maioria das quais constituindo formações secundárias em diferentes estádios sucessionais (Morellato \& Haddad 2000). Em face da fragmentação e da baixa disponibilidade de áreas primárias para a conservação da biodiversidade, florestas secundárias têm sido consideradas prioritárias para a conservação em várias partes da região tropical (Thomlinson et al. 1996; De Walt et al. 2003; Brearley et al. 2004).
As florestas secundárias apresentam modificações ao longo da sucessão, determinadas por fatores como a intensidade e frequiência de distúrbios (Martinez-Garza \& Howe 2003), a distância dessas áreas a uma fonte de propágulos (Uhl 1987; Uhl et al. 1998; Parrotta et al. 1997; Holl 1999), a forma de regeneração (Kennard et al. 2002; Kammesheidt 1999; Souza \& Batista 2004), a presença de espécies exóticas (Hooper et al. 2004), além de características do solo (Dalanesi et al. 2004). Áreas em estádios iniciais de sucessão freqüentemente apresentam baixa riqueza e são compostas por espécies generalistas e de distribuição geográfica ampla (Finegan 1996; Finegan \& Delgado 2000). Entre as características estruturais, o aumento de biomassa, volume, área basal, diâmetro e altura são apontadas como as principais

\footnotetext{
1 Parte da dissertação de Mestrado do primeiro Autor

2 Universidade Federal do Paraná, Setor de Ciências Biológicas, Departamento de Botânica, C. Postal 19.031, 81531-980 Curitiba, PR, Brasil

3 Autor para correspondência: dieterliebsch@yahoo.com.br
} 
mudanças durante o processo sucessional (Steininger 2000; Aide et al. 2000; Chinea 2002; Oliveira Filho et al.2004). Nos estádios iniciais há maior densidade de árvores, o que pode ser modificado rapidamente nos primeiros anos de sucessão (Finegan 1996). Com o aumento da idade das florestas, a estratificação vertical torna-se mais evidente, aumentando a complexidade estrutural da comunidade (Tabarelli \& Mantovani 1999a; Guilherme et al. 2004; Oliveira Filho et al. 2004).

Embora alguns trabalhos tenham abordado a estrutura da Floresta Atlântica em diferentes estádios da sucessão (Tabarelli \& Mantovani 1999a; Aidar et al. 2001; Siminiski et al. 2004), nenhum enfocou tais mudanças do ponto de vista quantitativo. Tal abordagem pode ser mais útil que levantamentos fitossociológicos tradicionais, por permitir comparações estruturais mais precisas. Informações sobre mudanças estruturais de florestas secundárias podem indicar se é possível predizer os caminhos da regeneração natural (Brearley et al. 2004).

O presente trabalho tem como objetivo examinar as diferenças estruturais de três florestas com idades distintas (20, 80 e 120 anos). As questões que norteiam a pesquisa são: Há mudanças florísticas e na diversidade a partir da floresta mais jovem (20 anos) até a mais antiga (120 anos)? Existem diferenças de abundância, riqueza e volume entre as três florestas? Há diferenças de densidade e volume entre as populações das espécies em comum nas três florestas?

\section{Material e métodos}

Local de estudo - O presente trabalho foi realizado na Reserva Natural Rio Cachoeira $\left(25^{\circ} 19^{\prime} 15^{\prime}\right.$ 'S e $45^{\circ} 42^{\prime} 24^{\prime \prime}$ ), Antonina, Paraná, pertencente à Sociedade de Pesquisa em Vida Selvagem e Educação Ambiental (SPVS). A reserva compreende 8.600 ha e inclui trechos da encosta da Serra do Mar e da planície litorânea, até a baía de Antonina, dentro dos limites da Área de Proteção Ambiental (APA) de Guaraqueçaba (Ferretti \& Britez 2006). O clima da região é chuvoso tropical sempre úmido (Af de Köeppen), com temperatura média de $21,1^{\circ} \mathrm{C}$, sem estação seca e geadas pouco freqüentes. A precipitação anual varia entre 2.500 e $3.000 \mathrm{~mm}$, com maior pluviosidade de janeiro a março (IPARDES 1991). A vegetação é do tipo Floresta Ombrófila Densa (IBGE 1992), ocupando as encostas até $500 \mathrm{~m}$ de altitude. As áreas de entorno da reserva sofreram exploração seletiva de madeira e desmatamento nas planícies para instalação de pastagens e culturas agrícolas. Atualmente, as comunidades do entorno da reserva vivem principalmente da pesca, cultivo de banana, arroz, gengibre, mandioca e hortaliças (Ferretti \& Britez 2006). Nas grandes propriedades, a atividade principal é a pecuária, principalmente de búfalos asiáticos.

Para o estudo foram escolhidos três sítios ao longo de um continuum de Floresta Ombrófila Densa Submontana (IBGE 1992), com até $800 \mathrm{~m}$ de distância entre si. A escolha considerou a idade aproximada de cada sítio, baseando-se no aspecto da vegetação retratado em aerofotos seqüenciais $(1952,1980 \mathrm{e}$ 2002), e no histórico de perturbação, obtido através de entrevistas com antigos moradores, cujas famílias eram radicadas na região há muitos anos. Desta forma, foram definidos três sítios com as idades aproximadas de 20, 80 e 120 anos desde o último distúrbio. O sítio com 20 anos encontrava-se sobre Cambissolo (R. Marques, comunicação pessoal) e desenvolveu-se em área de pastagem. O Sítio com 80 anos desenvolveuse em Argissolos (R. Marques, comunicação pessoal) onde anteriormente havia plantio de mandioca. $\mathrm{O}$ terceiro sítio, também sobre Cambissolo (R. Marques, comunicação pessoal), sofreu a retirada de madeira com valor comercial há aproximadamente 120 anos.

Coleta de dados - Foram estabelecidas parcelas de $10 \times 10 \mathrm{~m}$, com aproximadamente dois metros de distância entre si, onde foram amostrados todos os indivíduos com diâmetro à altura do peito $\geq 4,8 \mathrm{~cm}$. $\mathrm{O}$ número de parcelas em cada área foi determinado pela curva espécie/área (Müeller-Dombois \& Ellenberg 1974). Inicialmente foram amostradas 10 parcelas (unidades amostrais) em cada sítio e os dados foram utilizados para calcular a curva média de 10 simulações, utilizando o programa BioDiversity Professional. Após esta avaliação, os sítios com 80 e 120 anos não apresentaram uma assíntota e, portanto, outras cinco parcelas foram estabelecidas nesses sítios. Estas parcelas adicionais permitiram encontrar a suficiência no número de amostras. Assim, no sítio com 20 anos foram alocadas 10 parcelas e nos sítios com 80 e 120 anos foram alocadas 15 parcelas.

Cada indivíduo amostrado foi identificado, numerado e teve anotada a circunferência (posteriormente transformada em diâmetro), estimada a altura total (com uma régua comparativa, marcada a cada $50 \mathrm{~cm}$ ) e contado o número de caules (perfilhos). Coletas de material botânico foram feitas para a determinação taxonômica e para depósito como 
testemunho no Herbário do Departamento de Botânica da Universidade Federal do Paraná (UPCB). O enquadramento taxonômico das Angiospermas seguiu o Angiosperm Phylogeny Group (APG II 2003) e Tryon \& Tryon (1982) para as Pteridófitas.

Análises dos dados - A partir dos dados de altura total e diâmetro foi calculado o volume de cada indivíduo através da fórmula: $\mathrm{V}=\mathrm{d}^{2} * \mathrm{~h} * 0,4$ (onde, $\mathrm{V}=$ volume; $\mathrm{d}=$ diâmetro, $\mathrm{h}=$ altura e 0,4 é o fator de correção). Esta fórmula utiliza um fator de forma geral, desconsiderando as variações de forma de tronco existentes entre as espécies (Tilki \& Fisher 1998). Para caracterização estrutural, em cada sítio foram calculados os valores totais de abundância, densidade, riqueza, área basal, índice de diversidade de ShannonWiener (H') e índice de equabilidade de Pielou (J'). A similaridade florística entre os sítios foi calculada pelo coeficiente de Jaccard (Müeller-Dombois \& Ellenberg 1974). Diferenças das médias de abundância, riqueza e volume entre os sítios foram verificadas por Análise de Variância e teste Tukey-Kramer para múltiplas comparações (Zar 1999). Diferenças nas proporções de indivíduos com um ou mais perfilhos foram analisadas por teste Qui-Quadrado (Zar 1999). A existência de estratificação vertical nas comunidades foi avaliada a partir da distribuição dos indivíduos em classes de altura, cujas diferenças de freqüências entre os sítios foram avaliados com teste Qui-Quadrado (Zar 1999). As quatro classes de altura foram determinadas arbitrariamente. Em cada sítio foi analisada a participação de espécies exclusivas (somente ocorriam em um dado sítio) e comuns (ocorrência em mais que um sítio). Para tanto, compararam-se as proporções de espécies, indivíduos e do volume por teste QuiQuadrado (Zar 1999). Para espécies que ocorreram em mais de um sítio, as populações foram comparadas em densidade e volume médios, utilizando o teste $t$ (Zar 1999). Em todas as análises foi considerado um $\alpha=0,05$. Dados que não apresentaram distribuição normal foram transformados (log), o que ocorreu com as comparações de abundância, riqueza e volume médio entre os três sítios.

\section{Resultados}

No total foram amostrados 882 indivíduos e 102 espécies nas três áreas (Tab. 1). No sítio com 80 anos foram verificados os maiores valores absolutos de abundância, densidade e riqueza. No sítio com 120 anos foram verificados os maiores valores do índice de diversidade, equabilidade e área basal (Tab. 2). O coeficiente de similaridade de Jaccard (CSj) entre os

Tabela 1. Lista de espécies e respectivas abundâncias de três sítios de Floresta Atlântica na Reserva Natural Rio Cachoeira, Antonina, Paraná, Brasil.

\begin{tabular}{|c|c|c|c|}
\hline \multirow[t]{2}{*}{ Família/Espécie } & \multicolumn{3}{|c|}{ Sítio } \\
\hline & 20 anos & 80 anos & 120 anos \\
\hline \multicolumn{4}{|l|}{ ANACARDIACEAE } \\
\hline Tapirira guianensis Aubl. & & 4 & 2 \\
\hline \multicolumn{4}{|l|}{ ANNONACEAE } \\
\hline Guatteria australis A. St.-Hil. & & 2 & \\
\hline Rollinia emarginata Schltdl. & & & 1 \\
\hline R. sericea (R.E. Fr.) R.E. Fr. & & 2 & 2 \\
\hline \multicolumn{4}{|l|}{ APOCYNACEAE } \\
\hline Tabernaemontana catharinensis A. DC. & & 2 & \\
\hline \multicolumn{4}{|l|}{ AQUIFOLIACEAE } \\
\hline Ilex theazans Mart. & & 2 & \\
\hline Ilex sp. & & & 2 \\
\hline \multicolumn{4}{|l|}{ ARECACEAE } \\
\hline Astrocaryum aculeatissimum (Schott) Burret & & 5 & \\
\hline Euterpe edulis Mart. & & 22 & 22 \\
\hline \multicolumn{4}{|l|}{ ASTERACEAE } \\
\hline Vernonia puberula Less. & & & 1 \\
\hline \multicolumn{4}{|l|}{ BIGNONIACEAE } \\
\hline Tabebuia serratifolia (Vahl) G. Nicholson & & & 2 \\
\hline \multicolumn{4}{|l|}{ CANNELLACEAE } \\
\hline Capsicodendron dinisii (Schwacke) Occhioni & & & 1 \\
\hline
\end{tabular}


Tabela 1 (continuação)

\begin{tabular}{lrr}
\hline Família/Espécie & \multicolumn{2}{c}{ Sítio } \\
\cline { 2 - 3 } & 20 anos & 80 anos 120 anos \\
\hline
\end{tabular}

CELASTRACEAE

Maytenus alaternoides Reissek

CLUSIACEAE

Garcinia gardneriana (Planch. \& Triana) Zappi

CHRYSOBALANACEAE

Hirtella hebeclada Moric. ex DC.

CYATHEACEAE

Cyathea corcovadensis (Raddi) Domin

C. phalerata Mart.

ELAEOCARPACEAE

Sloanea guianensis (Aubl.) Benth.

EUPHORBIACEAE

Alchornea triplinervia (Spreng.) Müll. Arg.

A. glandulosa Poepp.

Maprounea brasiliensis A. St.-Hil.

Pera glabrata (Schott) Poepp. ex Baill.

FABACEAE

Andira fraxinifolia Benth.

Dalbergia frutescens (Vell.) Britton

Inga edulis Mart.

I. sessilis (Vell.) Mart.

Ormosia arborea (Vell.) Harms

Pterocarpus violaceus Vogel

Pseudopiptadenia warmingii (Benth.) G.P. Lewis \& M.P. Lima

Schizolobium parahyba (Vell.) S.F. Blake

Senna multijuga (Rich.) H.S. Irwin \& Barneby

Indeterminada

LAURACEAE

Nectandra leucantha Nees \& Mart.

N. membranacea (Sw.) Griseb.

Ocotea catharinensis Mez

O.laxa (Nees) Mez

O. elegans $\mathrm{Mez}$

LECYTHIDACEAE

Cariniana estrellensis (Raddi) Kuntze

MAGNOLIACEAE

Magnolia ovata (A. St. Hil.) Spreng.

MELASTOMATACEAE

Leandra dasytricha (A.Gray) Cogn.

Miconia cabucu Hohene

M. cinerascens Miq. var. robusta Wurdack

2

6

6

63

4

Tibouchina pulchra (Cham.) Cogn.

MELIACEAE

Cabralea canjerana (Vell.) Mart.

Guarea macrophylla Vahl

MONIMIACEAE

Mollinedia schottiana (Spreng.) Perkins

MORACEAE

Ficus insipida Willd.

MYRISTICACEAE

Virola bicuhyba (Schott ex Spreng.) Warb.

2

MYRSINACEAE

Myrsine coriacea (Sw.) R. Br. ex Roem. \& Schult.

MYRTACEAE

Calycorectes australis D. Legrand

Calyptranthes lucida Mart. ex DC.

C. strigipes O. Berg

10 
Tabela 1 (continuação)

\begin{tabular}{|c|c|c|c|}
\hline \multirow[t]{2}{*}{ Família/Espécie } & \multicolumn{3}{|c|}{ Sítio } \\
\hline & 20 anos & 80 anos & 120 anos \\
\hline Campomanesia reitziana $\mathrm{D}$. Legrand & & 1 & \\
\hline Eugenia catharinensis D. Legrand & & 1 & \\
\hline E. cf. cerasiflora Miq. & & 3 & \\
\hline E. cf. magnibracteolata Mattos \& D. Legrand & & & 2 \\
\hline E. melanogyna (D. Legrand) Sobral & & & 1 \\
\hline E. prasina $\mathrm{O}$. Berg & & 1 & \\
\hline E. stigmatosa DC. & & 2 & \\
\hline E. cf. uruguayensis Cambess & & & 2 \\
\hline Eugenia sp. 01 & & 1 & \\
\hline Eugenia sp. 02 & & 1 & \\
\hline Eugenia sp. 03 & & 1 & \\
\hline Eugenia sp. 04 & & & 2 \\
\hline Marlierea reitzii D. Legrand & & 1 & \\
\hline M. obscura O. Berg & & 4 & 12 \\
\hline M. sylvatica (Gardner) Kiaersk. & & 1 & \\
\hline M. tomentosa Cambess. & & & 45 \\
\hline Myrcia spectabilis DC. & & & 7 \\
\hline M. splendens (Sw.) DC. & & 1 & \\
\hline Myrceugenia miersiana (Gardner) D. Legrand \& Kausel & & & 1 \\
\hline Indeterminada & & & 1 \\
\hline \multicolumn{4}{|l|}{ NYCTAGINACEAE } \\
\hline Guapira opposita (Vell.) Reitz & & 14 & 9 \\
\hline \multicolumn{4}{|l|}{ PHYLLANTHACEAE } \\
\hline Hyeronima alchorneoides Allemão & & 40 & 2 \\
\hline \multicolumn{4}{|l|}{ PICRAMNIACEAE } \\
\hline Picramnia ramiflora Planch. & & & 1 \\
\hline \multicolumn{4}{|l|}{ PIPERACEAE } \\
\hline Piper cernuum Vell. & & & 1 \\
\hline \multicolumn{4}{|l|}{ QUIINACEAE } \\
\hline Quiina glaziovii Engl. & & 1 & 1 \\
\hline \multicolumn{4}{|l|}{ RUBIACEAE } \\
\hline Alibertia concolor (Cham.) K. Schum. & & & 1 \\
\hline Amaioua guianensis Aubl. & & 4 & \\
\hline Bathysa meridionalis L.B. Sm. \& Downs & & 80 & 2 \\
\hline Psychotria suterella Müll. Arg. & & 18 & 6 \\
\hline P. nuda (Cham. \& Schltdl.) Wawra & & 12 & 37 \\
\hline Randia armata (Sw.) DC. & & & 1 \\
\hline Rudgea jasminoides (Cham.) Müll. Arg. & & 38 & 8 \\
\hline \multicolumn{4}{|l|}{ RUTACEAE } \\
\hline Zanthoxylum rhoifolium Lam. & & 1 & \\
\hline Pilocarpus pauciflorus A. St.-Hil. & & 2 & 4 \\
\hline \multicolumn{4}{|l|}{ SALICACEAE } \\
\hline Casearia sylvestris $\mathrm{Sw}$. & & 4 & \\
\hline C. obliqua Spreng. & & 5 & 2 \\
\hline \multicolumn{4}{|l|}{ SAPINDACEAE } \\
\hline Allophyllus petiolatus Radlk. & & & 1 \\
\hline Cupania oblongifolia Mart. & 1 & 10 & 1 \\
\hline Matayba guianensis Aubl. & & 2 & \\
\hline M. juglandifolia Radlk. & & 6 & \\
\hline \multicolumn{4}{|l|}{ SAPOTACEAE } \\
\hline Chrysophyllum inornatum Mart. & & & 2 \\
\hline Chrysophyllum sp. & & 4 & \\
\hline Pouteria venosa (Mart.) Baehni & & 7 & 2 \\
\hline \multicolumn{4}{|l|}{ URTICACEAE } \\
\hline Cecropia pachystachya Trécul & 2 & & 9 \\
\hline Pourouma guianensis Aubl. & & & 3 \\
\hline \multicolumn{4}{|l|}{ VERBENACEAE } \\
\hline Citharexylum myrianthum Cham. & 1 & & \\
\hline
\end{tabular}


Tabela 2. Variáveis dos três sítios de Floresta Atlântica na Reserva Natural Rio Cachoeira, Antonina, Paraná, Brasil.

\begin{tabular}{lccc}
\hline Variáveis & \multicolumn{3}{c}{ Sítio } \\
\cline { 2 - 4 } & 20 anos & 80 anos & 120 anos \\
\hline Área amostrada $\left(\mathrm{m}^{2}\right)$ & 1.000 & 1.500 & 1.500 \\
$\begin{array}{l}\text { Abundância (indivíduos } \\
\quad \text { amostrados) }\end{array}$ & 189 & 451 & 242 \\
$\begin{array}{l}\left.\text { Densidade estimada (ind.ha }{ }^{-1}\right) \\
\text { Riqueza (n }{ }^{\circ} \text { espécies) }\end{array}$ & 1.890 & 3.006 & 1.600 \\
Índice de Shannon & 9 & 63 & 56 \\
Equabilidade de Pielou & 1,05 & 3,20 & 3,22 \\
Área basal (m ${ }^{2}$.ha $\left.{ }^{-1}\right)$ & 21,5 & 0,77 & 0,80 \\
& & 34,8 & 40,1 \\
\hline
\end{tabular}

sítios com 80 e 120 anos $(\mathrm{CSj}=29,21 \%)$ foi maior que entre os sítios de 20 e 80 anos $(\mathrm{CSj}=5,79 \%)$ e entre os de 20 e 120 anos $(\mathrm{CSj}=3,33 \%)$. A abundância média diferiu entre os sítios $\left(\mathrm{F}_{2 ; 39}=43,38 ; \mathrm{p}<0,0001\right)$, sendo que o sítio com 80 anos apresentou os maiores valores em relação aos demais, os quais não apresentaram diferenças entre si (Fig. 1A). A riqueza média foi maior $\left(\mathrm{F}_{2: 39}=51,95 ; \mathrm{p}<0,0001\right)$, no sítio com 80 anos que nos sítios com 20 e 120 anos (Fig. 1B). Os valores de volume foram maiores no sítio com 120 anos que no com 20 anos $\left(\mathrm{F}_{2: 39}=3,71 ; \mathrm{p}=0,03\right)$. O sítio com 80 anos não diferiu dos outros dois (Fig. 1C). A proporção de indivíduos apresentando perfilhos diferiu entre os três sítios $\left(\chi^{2}=18,02 ;\right.$ g.l. $=2 ; \mathrm{p}<$ $0,0001)$. A maior proporção de indivíduos com dois ou mais caules (em relação a apenas um) foi encontrada no sítio de 120 anos (15,2\%), seguida do de 20 anos $(13,5 \%)$ e o de 80 anos $(5,9 \%)$. A distribuição das classes de altura dos indivíduos apresentou proporções distintas nos três sítios $\left(\chi^{2}=107,02 ;\right.$ g.l. $\left.=6 ; p<0,0001\right)$. Em todos os sítios a maior proporção de indivíduos ocorreu na classe 1 (altura até $5 \mathrm{~m}$ ), mas no sítio com 120 anos a proporção de indivíduos das classes 3 e 4 (acima de 10,1 m) foi maior (Fig. 2).

As proporções de espécies comuns e exclusivas não apresentaram diferenças entre os três sítios $\left(\chi^{2}=0,0009 ;\right.$ g.l. $\left.=2 ; \mathrm{p}>0,05\right)$, mas diferiram em abundância $\left(\chi^{2}=29,26 ;\right.$ g.1. $\left.=2 ; \mathrm{p}<0,0001\right)$, com a maior proporção de indivíduos de espécies exclusivas no sítio com 120 anos, seguida pelos sítios com 20 e com 80 anos (Tab. 3). O volume das espécies exclusivas foi maior na floresta de 120, na floresta com 80 anos e menor no sítio com 20 anos (Tab. 3).

A maior parte das espécies que ocorreram em mais de um sítio não apresentou diferenças de densidade e volume médios (Tab. 4). Apenas as densidades de Rudgea jasminoides e Sloanea guianensis diminuíram do sítio de 80 anos para o sítio de 120 anos, enquanto que para Psychotria nuda este valor aumentou entre os sítios de 80 e 120 anos. Tibouchina pulchra teve decréscimo de densidade do sítio com 20 anos para o sítio com 80 anos. Euterpe edulis, Rudgea jasminoides e Sloanea guianensis, apresentaram volume médio maior no sítio com 120 anos do que no de 80 anos.

\section{Discussão}

As florestas em Antonina com 20, 80 e 120 anos apresentaram diferenças florísticas e estruturais, porém tais diferenças nem sempre ocorreram de forma
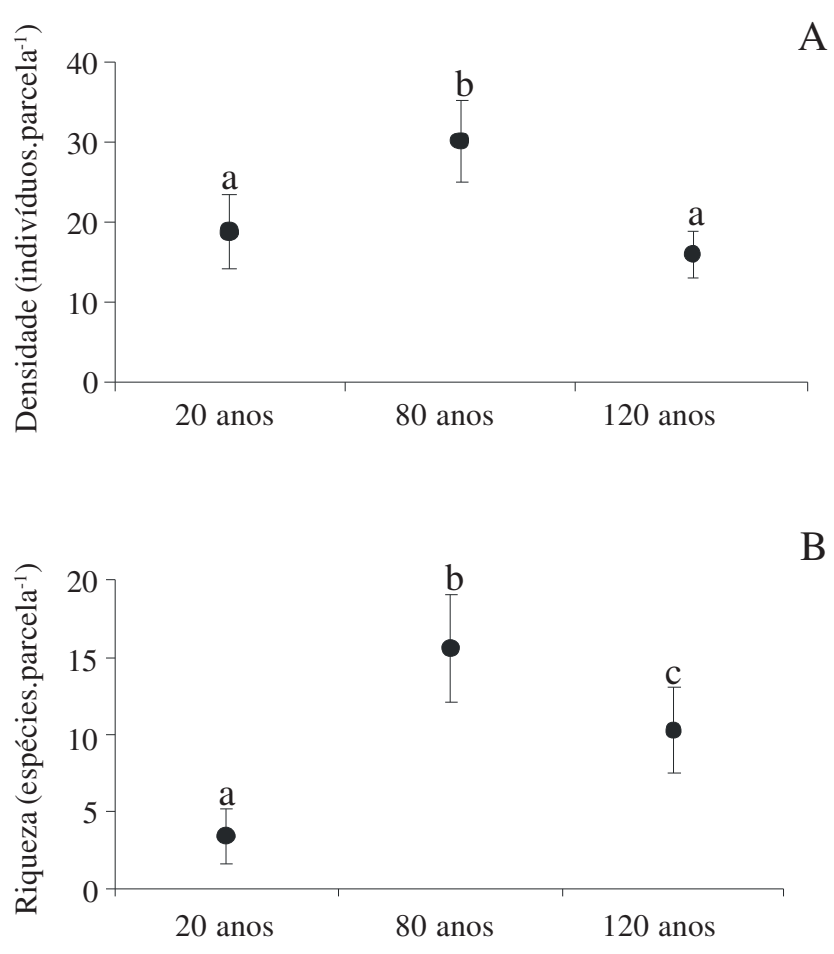

B

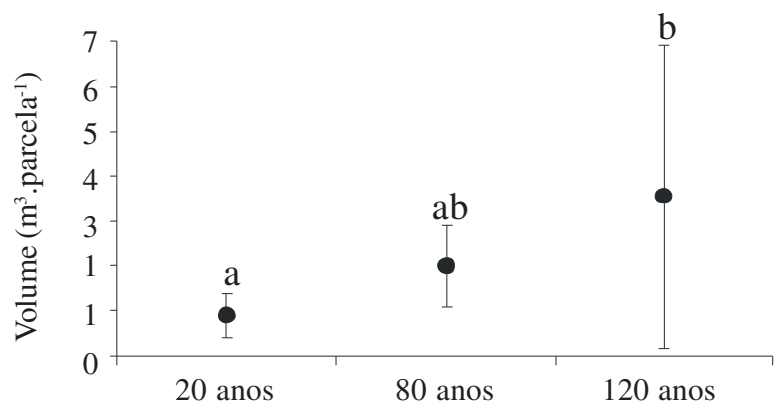

Figura 1. Valores médios ( \pm erro padrão) da abundância (A), riqueza (B) e volume (C) nos três sítios Floresta Atlântica na Reserva Natural Rio Cachoeira, Antonina, Paraná, Brasil. Sítios seguidos da mesma letra não diferem entre si (Tukey). 
gradativa, da floresta mais jovem para a mais antiga. As variações estruturais nestas florestas secundárias indicam a complexidade dos processos sucessionais

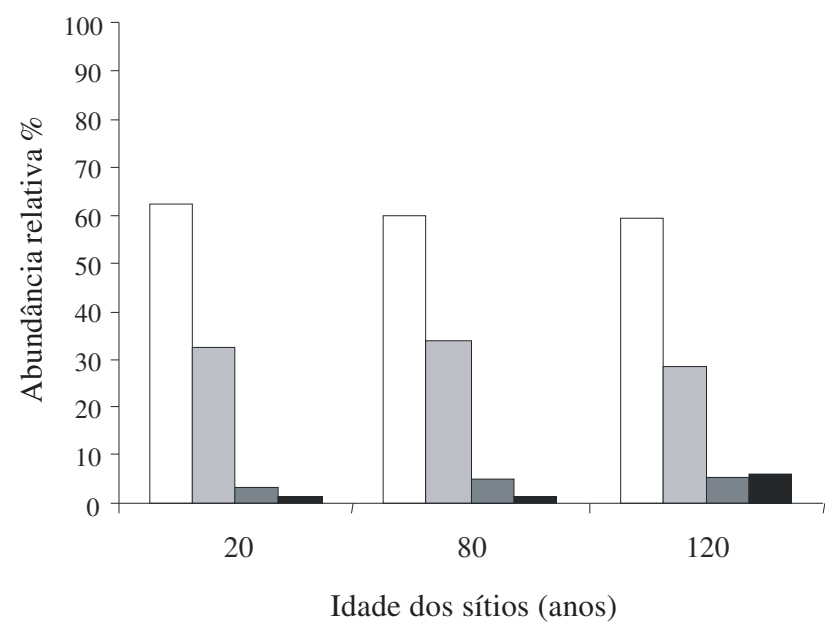

Figura 2. Distribuição dos indivíduos em classes de altura em três sítios de Floresta Atlântica na Reserva Natural Rio Cachoeira, Antonina, Paraná, Brasil. ( $\square=<5 \mathrm{~m} ; \quad \square=5,1$ a $10 \mathrm{~m} ; \quad \square=10,1 \mathrm{a}$ $15 \mathrm{~m} ; \boldsymbol{\square}=>15,1 \mathrm{~m})$. de regeneração natural de florestas tropicais.

A riqueza média das três florestas mostrou um aumento gradativo associado à idade da floresta. Embora o cálculo da média de riqueza possa subestimar as variações óbvias existentes na lista de espécies de cada sítio (resultando num número de espécies inferior ao real), os valores de riqueza total e índice de diversidade corroboram tal tendência. Baixa riqueza em florestas secundárias jovens é um padrão comum na região da Floresta Atlântica (Oliveira 2002;

Tabela 3. Contribuição das espécies exclusivas (\%) no total de riqueza, abundância e volume de cada sítio de Floresta Atlântica na Reserva Natural Rio Cachoeira, Antonina, Paraná, Brasil.

\begin{tabular}{cccc}
\hline \multirow{2}{*}{$\begin{array}{c}\text { Idade dos sítios } \\
\text { (anos) }\end{array}$} & \multicolumn{3}{c}{ Espécies exclusivas (\% do total) } \\
\cline { 2 - 4 } & Riqueza & Abundância & Volume \\
\hline 20 & 3,9 & 31,2 & 1,4 \\
80 & 28,1 & 19,5 & 6,8 \\
120 & 23,4 & 38,1 & 12,7 \\
\hline
\end{tabular}

Tabela 4. Densidade e volume médios das espécies comuns a mais de um sítio de Floresta Atlântica na Reserva Natural Rio Cachoeira, Antonina, Paraná, Brasil. (*) indica diferenças ( $<<0,05$, teste $T$ ) entre sítios com idades diferentes. (-): $\mathrm{n} \leq 3$.

\begin{tabular}{|c|c|c|c|c|c|c|}
\hline & \multicolumn{3}{|c|}{ Densidade média (ind.parcela ${ }^{-1}$ ) } & \multicolumn{3}{|c|}{ Volume médio $\left(\mathrm{m}^{3}\right.$.ind $\left.{ }^{-1}\right)$} \\
\hline & \multicolumn{3}{|c|}{ Sítio } & \multicolumn{3}{|c|}{ Sítio } \\
\hline & 20 anos & 80 anos & 120 anos & 20 anos & 80 anos & 120 anos \\
\hline Bathysa meridionalis & - & 5,33 & 0,13 & - & - & - \\
\hline Cecropia pachystachya & 0,20 & - & 0,60 & - & - & - \\
\hline Cabralea canjerana & - & 0,06 & 0,13 & - & - & - \\
\hline Casearia obliqua & - & 0,33 & 0,13 & - & - & - \\
\hline Cupania oblongifolia & 0,10 & 0,66 & 0,06 & - & - & - \\
\hline Cyathea phalerata & - & 0,40 & 0,40 & - & 0,016 & 0,030 \\
\hline Euterpe edulis & - & 1,40 & 1,40 & - & 0,019 & $0,030^{*}$ \\
\hline Guapira opposita & - & 0,93 & 0,60 & - & 0,049 & 0,021 \\
\hline Guarea macrophylla & - & 0,13 & 0,06 & - & - & - \\
\hline Hirtella hebeclada & - & 0,26 & 0,13 & - & - & - \\
\hline Hyeronima alchorneoides & - & 2,66 & 0,13 & - & - & - \\
\hline Machaerium uncinatum & - & 0,20 & 0,13 & - & - & - \\
\hline Marlierea obscura & - & 0,26 & 0,80 & - & 0,008 & 0,134 \\
\hline Mollinedia schottiana & - & 0,40 & 0,40 & - & 0,010 & 0,030 \\
\hline Nectandra membranacea & - & 0,53 & 0,13 & - & - & - \\
\hline Pera glabrata & 0,30 & 0,53 & - & 0,014 & 0,084 & - \\
\hline Pilocarpus pauciflorus & - & 0,13 & 0,26 & - & - & - \\
\hline Pouteria venosa & - & 0,46 & 0,13 & - & - & - \\
\hline Psychotria nuda & - & 0,80 & $2,46^{*}$ & - & 0,008 & 0,012 \\
\hline Psychotria suterella & - & 1,20 & 0,40 & - & 0,015 & 0,026 \\
\hline Quiina glaziovii & - & 0,06 & 0,06 & - & - & - \\
\hline Rollinia sericea & - & 0,13 & 0,13 & - & - & - \\
\hline Rudgea jasminoides & - & 2,53 & $0,53^{*}$ & - & 0,011 & $0,024^{*}$ \\
\hline Sloanea guianensis & - & 4,20 & $0,26 *$ & - & 0,052 & $2,533^{*}$ \\
\hline Tapirira guianensis & - & 0,26 & 0,20 & - & - & - \\
\hline Tibouchina pulchra & 12,40 & $0,13^{*}$ & - & - & - & - \\
\hline Virola bicuhyba & - & 0,06 & 0,13 & - & - & - \\
\hline
\end{tabular}


Siminiski et al. 2004). Em Antonina, os menores valores de riqueza foram registrados na floresta com 20 anos, onde Tibouchina pulchra foi a espécie mais abundante, formando densos agrupamentos (1240 ind.ha $\left.{ }^{-1}\right)$. Além disso, a utilização intensa deste sítio para pastagem pode ter influenciado o estabelecimento de plântulas de outras espécies, pois a presença de gramíneas exóticas pode ser barreira para o estabelecimento de plântulas (Hooper et al. 2004). Por outro lado, nos sítios com 80 e 120 anos, além de um tempo maior para a recuperação da floresta, o tipo de distúrbio (corte seletivo, na área com 120 anos) parece não ter causado tanto impacto, o que resultou em valores maiores de riqueza e diversidade. No entanto, ao contrário do esperado nos processos sucessionais (Clements 1936), a riqueza do sítio com 80 anos foi ligeiramente maior que a área com 120 anos. Provavelmente isto seja reflexo do fato de que no primeiro o sub-bosque era mais denso (devido a uma maior entrada de luz), o que elevou o número total de espécies. Além disso, o Argissolo do sítio com 80 anos tende a ser mais rico em nutrientes que o Cambissolo do sítio de 120 anos.

Associada às diferenças em riqueza houve dissimilaridade florística entre as três florestas, sendo esta mais evidente quando comparadas aos sítios de 20 e 120 anos. Com o desenvolvimento da sucessão, a heterogeneidade florística aumenta e a composição de espécies tende a se assemelhar às florestas secundárias maduras (Guariguata \& Ostertag 2001; De Walt et al. 2003). Com isso, aparentemente há uma rápida modificação florística nos primeiros anos da sucessão (demonstrado pela menor similaridade entre sítios de 20 e 80 anos) e uma lenta entrada de novas espécies nos períodos posteriores (maior semelhança entre os sítios de 80 e 120 anos).

As variações de densidade e de abundância não mostraram uma direção única na cronoseqüência, pois aumentaram a partir do sítio de 20 anos para o sítio de 80 anos e diminuíram desta para o sítio com 120 anos. A baixa densidade no sítio com 20 anos é esperada, devido ao baixo número de indivíduos compondo o subbosque. No sítio com 80 anos, a maior densidade deveu-se à presença de um dossel contínuo, com algumas espécies representadas por populações abundantes (principalmente Sloanea guianensis e Hyeronima alchorneoides) e de um sub-bosque bem desenvolvido (Psychotria suterella, P. nuda e Rudgea jasminoides). Já na floresta com 120 anos, embora o sub-bosque seja relativamente denso, o dossel apresenta indivíduos de grande porte (com DAP superior a $40 \mathrm{~cm}$ ), porém, com densidades baixas. Apesar das características do sub-bosque influenciarem fortemente nos aspectos estruturais, tais como densidade, diversidade e altura da floresta (Coelho et al. 2003; Guilherme et al. 2004), a ocorrência de variações de densidade em diferentes direções também em outras florestas secundárias (Aide et al. 2000; Chinea 2002; Brearley et al. 2004) sugere imprevisibilidade no processo sucessional e favorece a grande heterogeneidade florística, observada na Floresta Atlântica (Oliveira Filho \& Fontes 2000; Scudeller et al. 2001; Ferraz et al. 2004).

$\mathrm{O}$ volume (índice de biomassa) aumentou com o aumento da idade da floresta. Esta é uma das principais características da sucessão (Finergan 1996; Guariguata \& Ostertag 2001). O aumento na biomassa tende a ocorrer de maneira mais rápida nos primeiros 25 anos da sucessão (Aide et al. 2000), mas, em alguns casos, mesmo após um longo período de tempo (60 a 100 anos), a floresta tropical ainda não recupera totalmente a área basal e volume de florestas maduras (Saldarriaga et al. 1988; Turner et al. 1997). Em Antonina, na escala temporal abordada não foi possível concluir se houve retomada total da biomassa na floresta de 120 anos, mas o aumento da área basal, associado à menor densidade, indica aumento do porte dos indivíduos.

As maiores proporções de indivíduos com caules múltiplos no sítio com 120 anos e menor nos com 80 e 20 anos, indicam haver uma relação inversa entre o estabelecimento através de rebrotas e a idade das florestas. Isto contraria achados anteriores onde a relação encontrada era direta (Kammesheidt 1998; Simões \& Marques 2007). Por outro lado este maior número de caules no sítio com 120 anos pode estar relacionado ao tipo de distúrbio sofrido pela floresta (Miller \& Kauffman 1998).

Avaliando as comunidades em termos de espécies exclusivas e comuns, o sítio com 120 anos teve maior influência das espécies exclusivas em sua estrutura. Esse aumento da importância de espécies exclusivas em uma cronosequiência também foi observado anteriormente por Oliveira (2002). Isto deveu-se a presença de indivíduos de Marlierea tomentosa, Tabebuia serratifolia e Ocotea laxa, os quais ocorrem em áreas pouco perturbadas (Klein 1980), associado à presença de algumas espécies típicas de clareira, como Aegiphilla sellowiana, Vernonia puberula e Pourouma guianensis (Tabarelli \& Mantovani 1999b). Por outro lado, as espécies comuns, em geral, não mostraram diferenças significativas em 
suas populações nas três florestas. Portanto, as mudanças estruturais durante a sucessão podem ser explicadas em grande parte pelas características das espécies exclusivas.

\section{Agradecimentos}

À Sociedade de Proteção a Vida Selvagem e Educação Ambiental (SPVS), pela permissão e apoio logístico para o trabalho na Reserva Natural Rio Cachoeira; à Capes pela bolsa de mestrado a D. Liebsch; ao CNPq pela bolsa de produtividade em pesquisa a R. Goldenberg; a K.C. Cheug, J. Roper, M. Moura, F. Galvão e G. Durigan pelas sugestões ao manuscrito; a M. Sobral, M. Borgo e O.S. Ribas pelo auxilio na determinação de parte do material botânico. O trabalho é parte do projeto SOLOBIOMA, apoiado pelo CNPq e BMBF (German Federal Ministry of Education and Research).

\section{Referências bibliográficas}

Aidar, M.P.M.; Godoy, J.R.L.; Bergman, J. \& Joly, A.C. 2001. Atlantic Forest succesion over calcareous soil, Parque Estadual Turístico do Alto Ribeira - Petar, SP. Revista Brasileira de Botânica 24: 455-469.

Aide, T.M.; Zimmermman, J.K.; Pascarella, J.B.; Rivera, L. \& Marcano-Vega, H. 2000. Forest regeneration in a chronosequence of tropical abandoned pastures: implications for restoration ecology. Restoration Ecology 8: 328-338.

Angiosperm Phylogeny Group II. 2003. An update of the Angiosperm Phylogeny Group classification for the orders and families of flowering plants: APG II. Botanical Journal of the Linnean Society 141: 399-436.

Brearley, F.Q.; Prajadinata, S.; Kidd, P.S. \& Proctor, J., Suriantata, J.P. 2004. Structure and floristic of an old secondary rain forest in Central Kalimantan, Indonesia, and a comparison with adjacent primary forest. Forest Ecology and Management 195: 385-397.

Chinea, J.D. 2002. Tropical forest succession on abandoned farms in the Humacao Municipality of eastern Puerto Rico. Forest Ecology and Management 167: 195-207.

Clements, F.E. Nature and structure of the climax. 1936. Journal of Ecology 24: 252-284.

Coelho, R.F.R.; Zarin, D.J.; Miranda, I.S. \& Tucker, J.M. 2003. Análise florística e estrutural de uma floresta em diferentes estágios sucessionais no município de Castanhal, Pará. Acta Amazonica 33: 563-581.

Dalanesi, P.E.; Oliveira Fiho, A.T. \& Fontes, M.A.L. 2004. Flora e estrutura do componente arbóreo da floresta do Parque Ecológico Quedas do Rio Bonito, Lavras, MG, e correlações entre a distribuição das espécies e variáveis ambientais. Acta Botanica Brasilica 18: 737-757.
De Walt, S.J.; Maliakal, S.K. \& Denslow, J.S. 2003. Changes in vegetations structure and composition along a tropical forest chronosequence: implications for wildlife. Forest Ecology and Management 182: 139-151.

Ferraz, E.M.N.; Araújo, E.L. \& Silva, S.I. 2004. Floristic similarities between lowland and montane areas of Atlantic Coastal Forest in Northeastern Brazil. Plant Ecology 174: 59-70.

Ferretti, A.R. \& Britez, R.M. 2006. Ecological restoration, carbon sequestration and biodiversity conservation: The experience of the Society for Wildlife Research and Environmental Education (SPVS) in the Atlantic Rain Forest of Southern Brazil. Journal of Nature Conservation 14: 249-259.

Finegan, B. 1996. Pattern and process in neotropical secondary rain forest: the first 100 years of succession. Tree 11: 119-124.

Finegan, B. \& Delgado, D. 2000. Structural and floristic heterogeneity in a 30-year-old Costa Rican rain forest restored on pasture through natural secondary succession. Restoration Ecology 8: 380-393.

Guariguata, M.R. \& Ostertag. R. 2001. Neotropical secondary forest succession: changes in structural and functional characteristics. Forest Ecology and Management 148: 185-206.

Guilherme, F.A.G.; Morellato, L.P.C. \& Assis, M.A. 2004. Horizontal and vertical tree community structure in a lowland Atlantic Rain Forest, Southeastern Brazil. Revista Brasileira de Botânica 27: 725-737.

Holl, K.D. 1999. Factors limiting tropical rain forest regeneration in abandoned pasture: seed rain, seed germination, micro-climate and soil. Biotropica 31: 229-242.

Hooper, E.R.; Legendre, P. \& Condit, R. 2004. Factors affecting community composition of forest regeneration in deforested, abandoned land in Panamá. Ecology 85: 3313-3326.

IBGE, 1992. Manual Técnico da Vegetação Brasileira. Rio de Janeiro, Série Manuais Técnicos em Geociências, IBGE

IPARDES. 1991. Diagnóstico físico-ambiental da Serra do Mar: área Sul. Curitiba, IPARDES.

Kammesheidt, L. 1998. The role of tree sprouts in the restoration of stand structure and species diversity in tropical moist forest after slash-and-burn agriculture in Eastern Paraguay. Plant Ecology 139: 155-165.

Kammesheidt, L. 1999. Forest recovery by root suckers and above-ground sprouts after slash-and-burn agriculture, fire and logging in Paraguay and Venezuela. Journal of Tropical Ecology 15: 143-157.

Kennard, D.K.; Gould, K.; Putz, F.E.; Fredericksen, T.S. \& Morales, F. 2002. Effect of disturbance intensity on regeneration mechanisms in a tropical dry forest. Forest Ecology and Management 162:197-208.

Klein, R.M. 1980. Ecologia da flora e vegetação do Vale do Itajaí. Sellowia 32:165-389.

Leitão Filho, H.F. 1994. Diversity of arboreal species in Atlantic Rain Forest. Anais da Academia Brasileira de Ciências 66: 91-96. 
Martinez-Garza, C. \& Howe, H.F. 2003. Restoring tropical diversity: beating the time tax on species loss. Journal of Aplied Ecology 40: 423-429.

Miller, P.M. \& Kauffman, J.B. 1998. Seedling and sprout response to slash and burn agriculture in a tropical deciduos forest. Biotropica 30: 538-546.

Morellato, L.P.C. \& Haddad, C.F.B. 2000. The Brazilian Atlantic Forest. Biotropica 32: 786-792.

Müeller-Dombois, D. \& Ellenberg, H. 1974. Aims and methods of vegetation ecology. New York, John Wiley.

Myers, N.; Mittermeier, R.A.; Mittermeier, C.G.; Fonseca, G.A.B. \& Kent, J. 2000. Biodiversity hotspots for conservation priorities. Nature 403: 853-858.

Oliveira Filho, A.T.; Carvalho, D.A.; Vilela, E.A.; Curi, N. \& Fontes, M.A.L. 2004. Diversity and structure of the tree community of a fragment of tropical secondary Forest of the Brasilian Atlantic Forest domain 15 and 40 years after logging. Brasileira de Botânica 27: 685-701.

Oliveira Filho, A.T. \& Fontes, M.A.L. 2004. Patterns of floristic differentiation among Atlantic Forests in Southeastern Brazil and the influence of climate. Biotropica 32: 793-810.

Oliveira, R.R. 2002. Ação antrópica e resultantes sobre a estrutura e composição da Mata Atlântica na Ilha Grande, RJ. Rodriguésia 53: 33-58.

Parrotta, J.A.; Knowles, O.H. \& Wundesle, J.M. 1997. Development of floristic diversity in 10-years-old restoration forests on a bauxite mined site in Amazonia. Forest Ecology and Management 99: 21-42.

Saldarriaga, J.G.; West, D.C.; Thrp, M.L. \& Uhl, C. 1988. Long-term chronossequence of forest succession in the upper Rio Negro of Colombia and Venezuela. Journal of Ecology 76: 938-958.

Scudeller V.V.; Martins F.R. \& Shepherd G.J. 2001. Distribution and abundance of arboreal species in the Atlantic Ombrophylous Dense forest in Southeastern Brazil. Plant Ecology 152: 185-199.

Siminiski, A.; Mantovani, M.; Reis, M.S. \& Fantini, A.C. 2004. Sucessão florestal secundária no município de São Pedro de Alcântara, Litoral de Santa Catarina: estrutura e diversidade. Ciência Florestal 14: 21-33.
Simões, C.G. \& Marques, M.C.M. 2006. The role of sprouts in the restoration of Atlantic Rain Forest in southern Brazil. Restoration Ecology 15: 53-59.

Souza, F.M. \& Batista, J.L.F. 2004. Restoration of seasonal semideciduos Forest in Brazil: influence of age and restoration design on forest structure. Forest Ecology and Management 191: 185-200.

Steininger, M.K. 2000. Secondary forest structure and biomass following short and extended land-use in central and southern Amazonia. Journal of Tropical Ecology 16: 689-708.

Tabarelli, M. \& Mantovani, W. 1999a. A regeneração de uma Floresta Tropical Montana após corte e queima (São Paulo-Brasil). Revista Brasileira de Biologia 59: 239-250.

Tabarelli, M. \& Mantovani, W. 1999b. Clareiras naturais e a riqueza de espécies pioneiras em uma floresta Atlântica motana. Revista Brasileira de Biologia 59: 251-261.

Thomlinson, J.R.; Serrano, M.I.; López, T.; Aide, T.M. \& Zimmerman, J.K. 1996. Land-use dynamics in a postagriculture Puerto Rican landscape (1936-1988). Biotropica 28: 525-536.

Tilki, F. \& Fisher, R.F. 1998. Tropical leguminous species for acid soils: studies on plant form and growth in Costa Rica. Forest Ecology and Management 108: 175-792.

Tryon, R.M. \& Tryon, A.F. 1982. Ferns and allied plants with special reference to Tropical America. New York, Springer Verlag.

Turner, I.M.; Wong, Y.K.; Chew, P.T. \& Ibrahim, A.B. 1997. Tree species richness in primary and old secondary tropical forest in Singapore. Biodiversity Conservation 6: $537-543$.

Uhl, C. 1987. Factors controlling succession following slashand-burn agriculture in Amazonia. Journal of Ecology 75: $377-407$.

Uhl, C.; Buschbacher, R. \& Serrão, E.A.S. 1998. Abandoned pastures in eastern Amazonia: I. Patterns of plant succession. Journal of Ecology 76: 663-681.

Zar, J.H. 1999. Biostatistical analysis. New Jersey, PrenticeHall. 